\title{
STRUCTURE AND EMISSION LINE SPECTRUM OF AN X-RAY HEATED ACCRETION DISK IN AGN
}

\author{
YUAN-KUEN KO* and TIMOTHY R. KALLMAN \\ Laboratory for High Energy Astrophysics, NASA/Goddard Space Flight Center, USA.
}

\begin{abstract}
We investigate the structure of an X-ray heated accretion disk in active galactic nuclei. It is found that $\mathrm{X}$-ray heating can prevent the disk to be disrupted by its self-gravity under sufficient $\mathrm{X}$-ray heating. The disk size can be two orders of magnitute larger than that limited by self-gravity of the disk without $\mathrm{X}$-ray heating. An accretion disk corona will be formed by X-ray heating and can be a site for line emission. We present such emission line spectra which range from optical to hard $\mathrm{X}$-ray energies and compare with the observational data.
\end{abstract}

\section{Results}

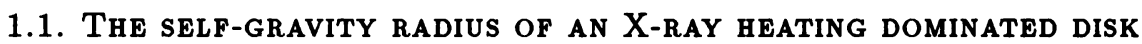

See Fig. 1. This is based on a disk where radiation pressure dominates gas pressure and electron scattering opacity dominates free-free opacity. (with $M=10^{8} M_{\odot}$ and $f \eta$ is the ratio of illuminating X-ray luminosity and $\dot{M} c^{2}$.)

\subsection{EMISSION SPECTRUM}

We made non-LTE and self-consistent calculations of the temperature, density, ionization, ion level populations and emitted spectrum in the accretion disk corona above the optically thick disk photosphere. Fig.2 shows the UV line ratios (relatiave to CIV $\lambda 1550$ ) compared with the observational data (Wu, Boggess \& Gull 1983, Laor et al. 1993).
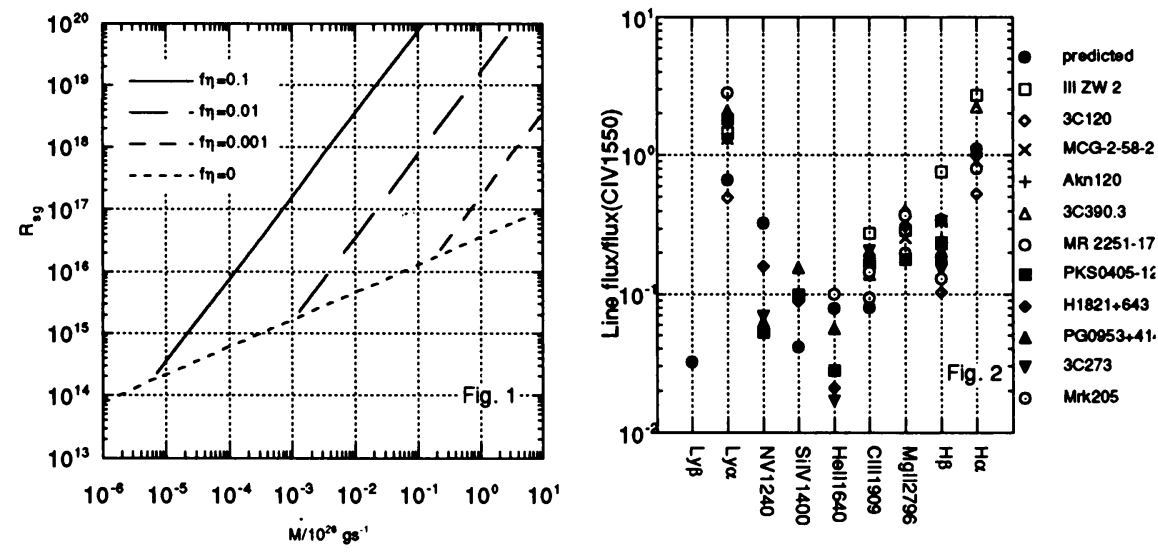

line

* Also Center for EUV Astrophysics, University of California, Berkeley, USA.

484

T. J.-L. Courvoisier and A. Blecha: Multi-Wavelength Continuum Emission of AGN, 484.

(c) 1994 IAU. Printed in the Netherlands. 